\title{
Hints of Dark Bosons
}

\section{A signal predicted for a type of dark matter appears in the spectra of ytterbium isotopes.}

By Katherine Wright

L xcitement is brewing among dark-matter researchers over signs that they may have detected the first signals of this elusive substance. In June, the XENON Collaboration reported seeing flashes of light that match those expected from a type of dark matter called dark bosons. Now two teams of researchers report results from another set of experiments also seeking dark bosons, with one of the teams finding hints consistent with this particle [1, 2].

Researchers have proposed a plethora of dark-matter candidates that explain astrophysical observations while conforming to the results of previous experiments. One of those candidates is the dark-matter boson, a particle that is predicted to interact weakly with ordinary matter. These dark bosons would be "virtually" exchanged between an atom's electrons and neutrons and induce tiny forces between them, thereby changing the atom's transition frequencies. Detecting this change was the aim of the two teams.

In their experiments, both teams measured the so-called isotope shift-the change in atomic spectra exhibited by

Credit: J. Hur/Massachusetts Institute of Technology isotopes of the same element. A group led by Vladan Vuletic of the Massachusetts Institute of Technology measured this shift between five ytterbium istotopes, while Michael Drewsen of Aarhus University in Denmark and colleagues measured the shift between five calcium ones.

The standard model predicts that, to first order, the isotope shifts should lie on a straight line on a "King" plot, a common method for representing the phenomenon. The Drewsen group's measurements fit with this prediction. The Vuletic group, however, reports a departure from predictions, finding a deviation from linearity with a statistical significance of 3 sigma. The researchers say that the deviation is consistent with a leading correction to the standard model's prediction, but it could also indicate the existence of dark bosons.

Katherine Wright is a Senior Editor for Physics.

\section{REFERENCES}

1. I. Counts et al., "Evidence for nonlinear isotope shift in $\mathrm{Yb}^{+}$search for new boson," Phys. Rev. Lett. 125, 123002 (2020).

2. C. Solaro et al., "Improved isotope-shift-based bounds on bosons beyond the standard model through measurements of the ${ }^{2} D_{3 / 2}-{ }^{2} D_{5 / 2}$ interval in Ca ${ }^{+}$," Phys. Rev. Lett. 125, 123003 (2020). 